\title{
Effectiveness of Short-Term Psychodynamic Psychotherapy in Preadolescents and Adolescents Affected by Psychiatric Disorders
}

\author{
Martina Maria Mensi ${ }^{1,2}$, Marika Orlandi ${ }^{1}$, Chiara Rogantini ${ }^{2}$, Renato Borgatti ${ }^{1,2}$, and Matteo Chiappedi ${ }^{1}$ \\ ${ }^{1}$ Child Neurology and Psychiatry Unit, IRCCS Mondino Foundation, Pavia, Italy \\ ${ }^{2}$ Child Neuropsychiatry Unit, Department of Brain and Behavioural Sciences, University of Pavia, Pavia, Italy
}

Objective Few studies have explored the effectiveness of short-term psychodynamic psychotherapy in children and adolescents. We aimed to investigate its efficacy in a heterogeneous group of young patients. We also wanted to check any relation between the improvement and patients' age, sex, or diagnostic category.

Methods We recruited a group of 123 patients (11 to 19 years old) with a diagnosis of psychiatric disorders confirmed by Kiddie Schedule for Affective Disorders and Schizophrenia (KSADS). All participants received eight sessions of psychodynamic psychotherapy ("Brief Individuation Psychotherapy" by Senise). They were assessed using the Clinical Global Impression (Severity at baseline, Improvement after treatment), and the Children's Global Assessment Scale (C-GAS).

Results We found a clinically meaningful improvement in most patients (CGI-I 3 or lower; 79 out of 123, 64.2\%) and a statistically significant improvement in the overall functioning (as measured by the C-GAS; $\mathrm{p}<0.001$ ). We found no effect of age or sex of the patient on results obtained; patients with an externalizing disorder had significantly poorer results.

Conclusion Our study, although lacking a control group, supports the possibility to use short-term psychodynamic psychotherapy in the treatment of psychiatric disorders in preadolescents and adolescents affected by psychiatric disorders.

Psychiatry Investig 2021;18(10):923-927

Keywords Adolescence; Brief psychotherapy; Evidence-based practice; Outcome study; Preadolescence; Psychiatric disorders.

\section{INTRODUCTION}

Psychodynamic psychotherapy has been considered not to be an evidence-based treatment due to lack of studies confirming its utility and to its excessive duration; this in turn prevented it to be included as recommended treatment for quite a long time, although over the years it proved to be an effective therapy in the treatment of many disorders ${ }^{1}$ and to have an effectiveness equal, if not superior to other manualized therapies ${ }^{2}$ as well as longer lasting effects. ${ }^{2,3}$ While there are many studies in the adult population that demonstrate the effectiveness of psychodynamic psychotherapy, the studies that include children and adolescents and the wide range of mental illnesses

Received: January 16, 2021 Revised: March 18, 2021

Accepted: June 20, 2021

$\triangle$ Correspondence: Matteo Chiappedi, MD, PhD

Child Neurology and Psychiatry Unit, IRCCS Mondino Foundation, Via Mondino 2, 27100 Pavia (PV), Italy

Tel: +390382 380285, Fax: +390382 380236

E-mail: matteo.chiappedi@mondino.it

(c) This is an Open Access article distributed under the terms of the Creative Commons Attribution Non-Commercial License (https://creativecommons.org/licenses/bync/4.0) which permits unrestricted non-commercial use, distribution, and reproduction in any medium, provided the original work is properly cited. that afflict them are far less common. ${ }^{4-6}$

Only recently, strong scientific evidence of the success of psychodynamic psychotherapy in the treatment of depression in childhood and adolescence has led to the inclusion of psychodynamic therapy in the English NICE Guidelines as a recommended treatment for moderate to severe depression. ${ }^{7}$ However, the committee has recognized that there are fewer studies supporting the effectiveness of psychodynamic psychotherapy than other types of therapy, such as individual cognitive-behavioural therapy (CBT), and therefore psychodynamic therapy is still considered an alternative option and not the first choice. The committee also expressed a recommendation to expand studies on the effectiveness of psychodynamic psychotherapy to a wide range of mental disorders affecting children and adolescents.

A problem which has been reported for years is the duration of original psychodynamic psychotherapy, which made it too long and expensive for the public health system. At present, a few studies support the effectiveness of short-term psychodynamic psychotherapy in the treatment of psychological disorders in adulthood, ${ }^{8,9}$ and even fewer studies have examined the 
effectiveness of short-term psychodynamic psychotherapy in developmental age. ${ }^{10,11}$

Considering that psychopathological disorders in childhood and adolescence are increasingly recognized ${ }^{12}$ and that $50 \%$ of pathologies in adulthood begin in earlier stages of life, especially in adolescence, ${ }^{13}$ it is necessary to use effective treatments as early as possible, to try and reach significant results in the short term but also to gain long-term benefits.

Given this framework, we aimed to investigate the efficacy of short-term psychodynamic psychotherapy in a heterogeneous group of young patients, using validated observational scales that measure the severity of disease, the overall functioning of patients and the improvement of their disease after the treatment. We also wanted to check if the improvement was related to patient's age, sex, or diagnostic category.

We hypothesized that taking part in a cycle of psychodynamic psychotherapy, even of short duration, could produce an improvement in the overall functioning of preadolescent and adolescent patients and an improvement in their symptoms. Moreover, we would expect patients diagnosed with internalizing disorders to better respond to psychotherapeutic treatment, independently of age or sex., ${ }^{3,14,15}$

\section{METHODS}

\section{Participants}

We recruited 123 preadolescents and adolescents (61 females and 62 males; mean age $=14.9$ years, $\mathrm{SD}=1.79 ; \mathrm{SEM}=0.16$; age range: 11-19 years, extremes included) seen at the Child Neurology and Psychiatry Unit of the IRCCS Mondino Foundation in Pavia, Italy, between 2017 and 2018.

Patients younger than 11 years old or older than 19 years old, with insufficient comprehension of the Italian language or with an established diagnosis of intellectual disability were excluded. In accordance with guidelines and recent evidence, patients taking drug therapy were not excluded. In our sample, 3.33\% assumed benzodiazepines, $3.33 \%$ antidepressants, $3.33 \%$ antipsychotic drugs, $0.8 \%$ mood stabilizers.

\section{Procedure}

We collected data within a larger project named "Study of prognosis and possible predictors of outcome in a population of adolescent patients with psychosis, attenuated psychosis and not psychotic psychiatric disorders," authorized in 2017 by the Ethical Committee of Policlinico San Matteo in Pavia, Italy (P20170028892). We followed the code of good ethical practice and the ethical standards of The Declaration of Helsinki (1964) and its later amendments. All patients and their families gave their written informed consent to participate in the study and were free to retreat in any moment. We informed participant that data would have been used for research purposes only and we would have anonymized them to protect privacy.

We started with an evaluation phase which included meetings with patient and his/her family, to collect family and medical history through clinical interviews and diagnostic analysis. At baseline $\left(\mathrm{T}_{0}\right)$ clinician or psychotherapist compiled two scales to assess the severity of the disease (CGI-S) and the patient's global functioning (C-GAS). After the assessment, every participant underwent a cycle of manualized psychotherapy based on the model of "Brief Individuation Psychotherapy" by Tommaso Senise. ${ }^{16}$ Treatment followed a protocol focusing on the separation-individuation process. The first two sessions were dedicated to the investigation of the patient's relationships with siblings and adults, mainly focusing on conflicts relating to the process of definition of one's own identity with a psychological separation especially from the parents. The following sessions were devoted to the exploration and elaboration of the more significant psychopathological nuclei. The major goal of the treatment was to allow the patient to develop an empathic identification with the therapist (mirror identification) in order to reach a progressively more adaptive understanding of his/ her difficulties and ultimately to overcome them. ${ }^{17}$ Treatment was administered by expert psychotherapists, with four-year specialization in psychotherapy and experience in the treatment of youth and adolescents. Patients with psychotic symptoms during therapy had no positive symptoms. After eight weekly sessions psychotherapy of one hour each $\left(\mathrm{T}_{1}\right)$, the same clinician or psychotherapist who took charge the patient compiled a scale to indicate improvement (CGI-I) and recompiled the scale that indicates his/her global functioning (C-GAS).

\section{Measures}

To confirm the diagnosis, psychologists or child neuropsychiatrists conducted with both children/adolescents and their parents/caregivers the Kiddie Schedule for Affective Disorders and Schizophrenia (KSADS), ${ }^{18}$ a diagnostic semi-structured interview based on DSM criteria for the assessment of psychopathological disorders in children and adolescents. We grouped the diagnoses from KSADS in five categories inspired by ASEBA system: no diagnosis, psychotic disorders, internalizing disorders (depressive disorders, anxiety disorders, OCD), externalizing disorders (ADHD, oppositional defiant disorder, conduct disorder), mixed disorders.

To assess the progress of patients' conditions, clinician or psychotherapist filled in the:

\section{Clinical Global Impression (CGI) $)^{19,20}$}

One of the most widely used rapid assessment scales in psychiatry, both in the field of research and clinic. It measures the severity of the disease (CGI-Severity), the improvement or over- 
all change of the patient (CGI-Improvement), and the therapeutic response. The evaluation of severity of the patient's conditions must be expressed at each visit, including the first one, assigning scores from 1 (normal, not at all ill) to 7 (among the most extremely ill patients). The improvement assessment must be made during every visit after baseline choosing a score from 1 (very much improved) to 7 (very much worse). Each section of this scale is assessed separately and does not have an overall score.

\section{Children's Global Assessment Scale (C-GAS) ${ }^{21,22}$}

This is a useful scale for the evaluation of the patient's overall functioning. It provides a measure of the overall severity coded in 10 ranges from the lowest 1-10 (need for constant supervision as a consequence of self-destructive behaviour or dominated by important aggression, or a serious impairment of reality analysis, communication, cognitive, emotional or personal hygiene level) to the highest 91-100 (superior functioning in every area of daily life, both at home and at school and in relations with peers, involvement in a wide range of activities, presence of numerous interests, daily concerns constantly under control, good school performance, absence of symptoms). This scale is simple to compile and is characterized by excellent psychometric properties. ${ }^{21}$

Evaluators shared assessment results with clinician or psychotherapist prior to treatment. At $\mathrm{T}_{0}$ clinician or psychotherapist compiled CGI-Severity and CGAS. After eight sessions of psychotherapy ${ }^{17}\left(\mathrm{~T}_{1}\right)$, the same clinician or psychotherapist who oversaw the patient filled in CGI-Improvement and a new CGAS.

\section{Data analysis}

Analyses were conducted using IBM SPSS Version 21 for Windows (IBM Corp., Armonk, NY, USA). After assessing descriptive statistics for each variable, a preliminary one-sample Kolmogorov-Smirnov test for normality was computed. Paired sample t-test was used to assess differences for normally distributed variables comparing the same subjects before and after treatment, while Spearman correlation coefficient was used to assess correlations involving ordinal variables.

\section{RESULTS}

\section{Descriptive statistics}

The sample included 123 preadolescents and adolescents (49.59\% females) from 11 to 19 years old, with a mean age of 14.9 ( $\mathrm{SD}=1.79$; $\mathrm{SEM}=0.16)$. No patient dropped out of research before the end. Table 1 shows means, SDs and SEMs for all variables.

The most frequent diagnosis within the sample was inter- nalizing disorders, and most participants received an evaluation of disease severity from 3 to 5 at baseline (Table 2).

\section{First objective}

Table 3 shows that most of participants received a score of

Table 1. Descriptive analysis for global functioning (at baseline and posttreatment) and severity of disease (at baseline and posttreatment)

\begin{tabular}{lcccc}
\hline & C-GAS $_{\mathrm{T} 0}$ & CGI-S $_{\mathrm{T} 0}$ & C-GAS $_{\mathrm{T} 1}$ & CGI-I $_{\mathrm{T} 1}$ \\
\hline $\mathrm{M}$ & 56.41 & 3.98 & 62.78 & 2.94 \\
$\mathrm{SD}$ & 17.05 & 1.32 & 17.30 & 1.09 \\
$\mathrm{SEM}$ & 1.54 & 0.12 & 1.56 & 0.10 \\
\hline
\end{tabular}

C-GAS, Children's Global Assessment Scale; CGI-S, Clinical Global Impression-Status; CGI-I, Clinical Global Impression-Improvement; M, mean; SD, standard deviation; SEM, standard error of the mean

Table 2. Frequencies and percentages of diagnosis and disease severity scores at $T_{0}$

\begin{tabular}{lc}
\hline & $\begin{array}{c}\text { Frequency } \\
(\mathrm{N}=123), \%\end{array}$ \\
\hline Diagnosis & \\
$0=$ No diagnoses & $0(0)$ \\
$1=$ Psychotic disorders & $5(4.1)$ \\
2=Internalizing disorders & $70(56.9)$ \\
$3=$ Externalizing disorders & $20(16.3)$ \\
$4=$ Mixed disorders & $28(22.8)$ \\
CGI-S T & \\
$0=$ Not assessed & $0(0)$ \\
1=Normal, not at all ill & $4(3.2)$ \\
2=Borderline mentally ill & $13(10.6)$ \\
$3=$ Mildly ill & $22(17.9)$ \\
$4=$ Moderately ill & $46(37.4)$ \\
$5=$ Markedly ill & $21(17.1)$ \\
6=Severely ill & $14(11.4)$ \\
$7=$ Among the most extremely ill patients & $3(2.4)$ \\
\hline CGI-S, Clinical Global Impression-Status &
\end{tabular}

Table 3. Frequencies and percentages of improvement scores at $T_{1}$

\begin{tabular}{lc}
\hline & Frequency $(\mathrm{N}=123), \%$ \\
\hline CGI-I & $13(10.6)$ \\
1=Very much improved & $26(21.1)$ \\
2=Much improved & $40(32.5)$ \\
3=Minimally improved & $39(31.7)$ \\
$4=$ No change & $5(4.1)$ \\
5=Minimally worse & $0(0)$ \\
6=Much worse & $0(0)$ \\
$7=$ Very much worse &
\end{tabular}

CGI-I, Clinical Global Impression-Improvement 


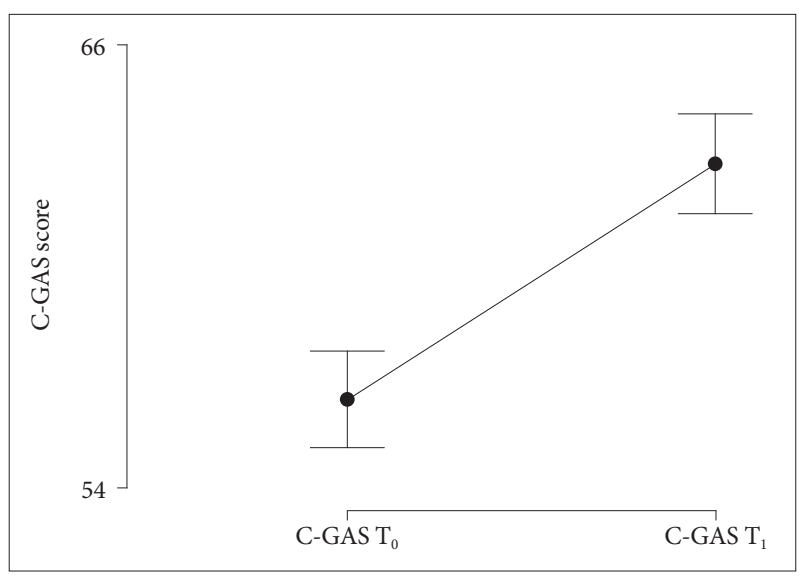

Figure 1. C-GAS scores improvement from $T_{0}$ to $T_{1}$. C-GAS, Children's Global Assessment Scale.

illness improvement from 3 to 1 after treatment, showing improvement in 79 out of 123 patients (64.2\%).

There was a statistically significant improvement in the overall functioning of patients after psychotherapeutic treatment measured with C-GAS [t $(122)=-6.79, \mathrm{p}<0.001]$ (Figure 1).

\section{Second objective}

We found no significant correlations between diagnosis and age or sex. In the same way, improvement score (CGI-I) did not correlate with age or sex. We found however a significant correlation between diagnostic category and improvement $(r=$ $0.185, \mathrm{p}=0.041$ ), as patients diagnosed with externalizing problems seemed to improve significantly less than the others (average CGI-I score $3.70 \pm 0.73$ for externalizing disorders, versus an average of the other groups being $2.79 \pm 1.06$ without statistically significant differences between the other diagnostic groups).

\section{DISCUSSION}

In our sample, internalizing disorders were, as also found in the Italian and international literature, those most diagnosed, followed by externalizing problems, mixed ones, and psychotic disorders. ${ }^{23-27}$

Unlike other studies that found differences in prevalence due to age and gender, ${ }^{12}$ our sample does not present any significant correlation between KSADS diagnosis and age, and neither between KSADS diagnosis and gender. This can be explained by the choice not to divide the sample between preadolescents and adolescents, but also by the decision to combine the diagnoses into fewer broader diagnostic categories according to KSADS and ASEBA system (internalizing, externalizing, mixed, and psychotic disorders).

Moreover, our first hypothesis was confirmed. A group of youth of various ages was considered so as not to deprive any- one of treatment. To address the inhomogeneity of the sample, statistical analyses were conducted for paired samples, and, according to our findings, most of participants had an improvement in their mental health condition from minimal to considerable after treatment. In line with this, the scale used to evaluate the global functioning of patients (C-GAS) showed a statistically significant improvement in the overall functioning, as previously proven in adult patients affected by common mental disorders. ${ }^{8,9}$

Regarding the second hypothesis, despite some studies that stated that younger children, ${ }^{28}$ and females ${ }^{29}$ appear to benefit more from psychodynamic psychotherapy, according to our data the improvement in mental health condition and in the global functioning after short-term psychodynamic psychotherapy was independent of both age and sex of the patient. Our data seem to confirm, in line with previous studies, $3,14,15$ that patients who seem to benefit most from short-term psychodynamic psychotherapy are those diagnosed with internalizing disorders, mixed problems and psychotic disorders. Patients with internalizing disorders can more easily build a therapeutic alliance and are more willing to reflect on their emotional states. In fact, as expected from existing literature, ${ }^{14}$ patients suffering from externalizing disorders are those who showed the less enhancement, because they have more difficulties in building a therapeutic alliance and/or take longer to do so because of their severe behavioural impairment. Moreover, because few patients were taking drug treatment, we can assume that the changes seen after treatment are not mainly related to pharmacological interventions.

This study has limitations, in fact future research could include participants from other regions and a control group in order to confirm our findings and to test their strength. In this study, we could not have a control group because the setting was that of a research-action study (i.e., we had to balance research with clinical practice). Furthermore, we hope that future studies could also compare the evidence collected by clinicians with the perception of change from the point of view of patients and their families, e.g., using self-report questionnaires. The fact that our sample was quite heterogeneous represents a limitation on one hand, but also a positive aspect as it resembles real-life clinical practice. Moreover, future research should include a longer follow up to assess stability of obtained changes.

\section{Availability of Data and Material}

The datasets generated or analyzed during the current study are available in the Zenodo repository, DOI: 10.5281/zenodo.4956736.

\section{Conflicts of Interest}

The authors have no potential conflicts of interest to disclose. 


\section{Author Contributions}

Conceptualization: Martina Maria Mensi, Matteo Chiappedi. Data curation: Marika Orlandi, Chiara Rogantini. Formal analysis: Matteo Chiappedi. Investigation: Marika Orlandi, Chiara Rogantini. Methodology: Martina Maria Mensi, Matteo Chiappedi, Renato Borgatti. Project administration: Marika Orlandi, Chiara Rogantini. Supervision: Martina Maria Mensi, Matteo Chiappedi, Renato Borgatti. Writing_original draft: Marika Orlandi, Chiara Rogantini. Writing — review \& editing: Marika Orlandi, Chiara Rogantin, Matteo Chiappedi. All authors have read and agreed to the present version of the manuscript.

\section{ORCID iDs}

Martina Maria Mensi

Marika Orlandi

Chiara Rogantini

Renato Borgatti

Matteo Chiappedi

https://orcid.org/0000-0002-9583-0554 https://orcid.org/0000-0003-2783-5262 https://orcid.org/0000-0002-5297-5825 https://orcid.org/0000-0001-8165-4994 https://orcid.org/0000-0002-2682-5006

\section{Funding Statement}

None

\section{REFERENCES}

1. Shedler J. The efficacy of psychodynamic psychotherapy. Am Psychol 2010;65:98-109.

2. Leichsenring F, Steinert C. The Efficacy of Psychodynamic Psychotherapy: An Up-to-Date Review. In: Kealy D, Ogrodniczuk J, Editors. Contemporary Psychodynamic Psychotherapy: Evolving Clinical Practice. London: Academic Press; 2019, p.49-74.

3. Fonagy P. The effectiveness of psychodynamic psychotherapies: an update. World Psychiatry 2015;14:137-150.

4. Weisz JR, Weiss B, Han SS, Granger DA, Morton T. Effects of psychotherapy with children and adolescents revisited: a meta-analysis of treatment outcome studies. Psychol Bull 1995;117:450-468.

5. Palmer R, Nascimento LN, Fonagy P. The state of the evidence base for psychodynamic psychotherapy for children and adolescents. Child Adolesc Psychiatr Clin N Am 2013;22:149-214.

6. Midgley N, Kennedy E. Psychodynamic psychotherapy for children and adolescents: a critical review of the evidence base. J Child Psychother 2011;37:232-260.

7. National Institute for Health and Care Excellence (NICE). Depression in children and young people: identification and management | Guidance |. London: NICE; 2019.

8. Abbass AA, Kisely SR, Town JM, Leichsenring F, Driessen E, De Maat S, et al. Short-term psychodynamic psychotherapies for common mental disorders. Cochrane Database Syst Rev 2014;(7):CD004687.

9. Lewis AJ, Dennerstein M, Gibbs PM. Short-term psychodynamic psychotherapy: review of recent process and outcome studies. Aust N Z J Psychiatry 2008;42:445-455.

10. Abbass AA, Rabung S, Leichsenring F, Refseth JS, Midgley N. Psychodynamic psychotherapy for children and adolescents: a meta-analysis of short-term psychodynamic models. J Am Acad Child Adolesc Psychiatry 2013;52:863-875.

11. Gatta M, Miscioscia M, Svanellini L, Spoto A, Difronzo M, de Sauma M, et al. Effectiveness of brief psychodynamic therapy with children and adolescents: an outcome study. Front Pediatr 2019;7:501.

12. Merikangas KR, Nakamura EF, Kessler RC. Epidemiology of mental disorders in children and adolescents. Dialogues Clin Neurosci 2009; 11:7-20.

13. Patel V, Flisher AJ, Hetrick S, McGorry P. Mental health of young people: a global public-health challenge. Lancet 2007;369:1302-1313.

14. Fonagy P, Target M. The efficacy of psychoanalysis for children with disruptive disorders. J Am Acad Child Adolesc Psychiatry 1994;33:4555.

15. Muratori F, Picchi L, Casella C, Tancredi R, Milone A, Patarnello MG. Efficacy of brief dynamic psychotherapy for children with emotional disorders. Psychother Psychosom 2002;71:28-38.

16. Aliprandi M, Pelanda E, Senise T. Psicoterapia Breve Di Individuazione. Milano: Mimesis; 2014.

17. Balottin U, Ferri M, Racca M, Rossi M, Rossi G, Beghi E, et al. Psychotherapy versus usual care in pediatric migraine and tension-type headache: a single-blind controlled pilot study. Ital J Pediatr 2014;40.

18. Kaufman J, Schweder AE. The Schedule for Affective Disorders and Schizophrenia for School-Age Children: Present and Lifetime Version (K-SADS-PL). In: Hilsenroth M, Segal D, Editors. Comprehensive Handbook of Psychological Assessment. Hoboken, New Jersey: John Wiley \& Sons Inc; 2004, p.247-255.

19. Guy W. ECDEU Assessment Manual for Psychopharmacology. Rockville, MD: U.S. Department of Health, Education, and Welfare; 1976.

20. Berk M, Ng F, Dodd S, et al. The validity of the CGI severity and improvement scales as measures of clinical effectiveness suitable for routine clinical use. J Eval Clin Pract 2008;14:979-983.

21. Shaffer D, Gould MS, Brasic J, Callaly T, Campbell S, Bernardo M, et al. A Children's Global Assessment Scale (CGAS). Arch Gen Psychiatry 1983;40:1228-1231.

22. Masi G, Milone A, Scrinzi G, Mucci M, Viglione V, Bruni G, et al. Lithium treatment in bipolar adolescents: a follow-up naturalistic study. Neuropsychiatr Dis Treat 2018;14:2749-2753.

23. Frigerio A, Rucci P, Goodman R, Ammaniti M, Carlet O, Cavolina P, et al. Prevalence and correlates of mental disorders among adolescents in Italy: the PrISMA study. Eur Child Adolesc Psychiatry 2009;18:217226.

24. Erskine HE, Baxter AJ, Patton G, Moffitt TE, Patel V, Whiteford HA, Scott JG. The global coverage of prevalence data for mental disorders in children and adolescents. Epidemiol Psychiatr Sci 2017;26:395-402.

25. Merikangas KR, He JP, Burstein M, Swanson SA, Avenevoli S, Cui L, et al. Lifetime prevalence of mental disorders in U.S. adolescents: results from the national comorbidity survey replication-adolescent supplement (NCS-A). J Am Acad Child Adolesc Psychiatry 2010;49:980-989.

26. La Maison C, Munhoz TN, Santos IS, Anselmi L, Barros FC, Matijasevich A. Prevalence and risk factors of psychiatric disorders in early adolescence: 2004 Pelotas (Brazil) birth cohort. Soc Psychiatry Psychiatr Epidemiol 2018;53:685-697.

27. Lawrence D, Johnson S, Hafekost J, Boterhoven De Haan K, Sawyer M, Ainley J, et al. The Mental Health of Children and Adolescents?: Report on the Second Australian Child and Adolescent Survey of Mental Health and Wellbeing. Canberra, ACT: Department of Health; 2015.

28. Odhammar F, Sundin EC, Jonson M, Carlberg G. Children in psychodynamic psychotherapy: changes in global functioning. J Child Psychother 2011;37:261-279.

29. Deakin EK, Nunes MLT. Effectiveness of child psychoanalytic psychotherapy in a clinical outpatient setting. J Child Psychother 2009;35:290301. 